\title{
THE INTERCONNECTIONS OF THE LHC CRYOMAGNETS
}

\author{
A. Jacquemod, A. Poncet, B. Skoczen, J.Ph. Tock, CERN, Geneva, Switzerland
}

\section{Abstract}

The main components of the LHC, the next world-class facility in high-energy physics, are the twin-aperture highfield superconducting cryomagnets to be installed in the existing 26.7-km long tunnel. After installation and alignment, the cryomagnets have to be interconnected. The interconnections must ensure the continuity of several functions: vacuum enclosures, beam pipe image currents (RF contacts), cryogenic circuits, electrical power supply, and thermal insulation.

In the machine, about 1700 interconnections between cryomagnets are necessary. The interconnections constitute a unique system that is nearly entirely assembled in the tunnel. For each of them, various operations must be done: TIG welding of cryogenic channels $(\approx 50000$ welds $)$, induction soldering of main superconducting cables $(\approx 10000$ joints $)$, ultrasonic welding of auxiliary superconducting cables $(\approx 20000$ welds), mechanical assembly of various elements, and installation of the multi-layer insulation $\left(\approx 200000 \mathrm{~m}^{2}\right)$. Defective junctions could be very difficult and expensive to detect and repair. Reproducible and reliable processes must be implemented together with a strict quality control.

The interconnection activities are optimized taking into account several constraints: limited space availability, tight installation schedule, high level of quality, high reliability and economical aspects.

In this paper, the functions to be fulfilled by the interconnections and the various technologies selected are presented. Quality control at different levels (component/ interconnect, subsystem, system) is also described. The interconnection assembly sequences are summarized. Finally, the validation of the interconnection procedures is presented, based in particular on the LHC prototype cell assembly (STRING2).

\section{INTRODUCTION}

The interconnections of the twin-aperture high-field superconducting cryomagnets will be carried out in the tunnel. They must ensure the continuity of several functions: vacuum enclosures, beam pipes, beam screen image currents (RF contacts), cryogenic circuits, electrical power supply, thermal insulation, etc. For the whole LHC machine, about 1700 interconnections between cryomagnets have to be carried out. Every $108 \mathrm{~m}$, the chain of superconducting magnets is connected to the cryogenic distribution line by so called "jumpers". The continuity of vacuum enclosures, cryogenic circuits and thermal insulation must be ensured through the jumpers. The two basic types of interconnection may be seen in Fig. 1.

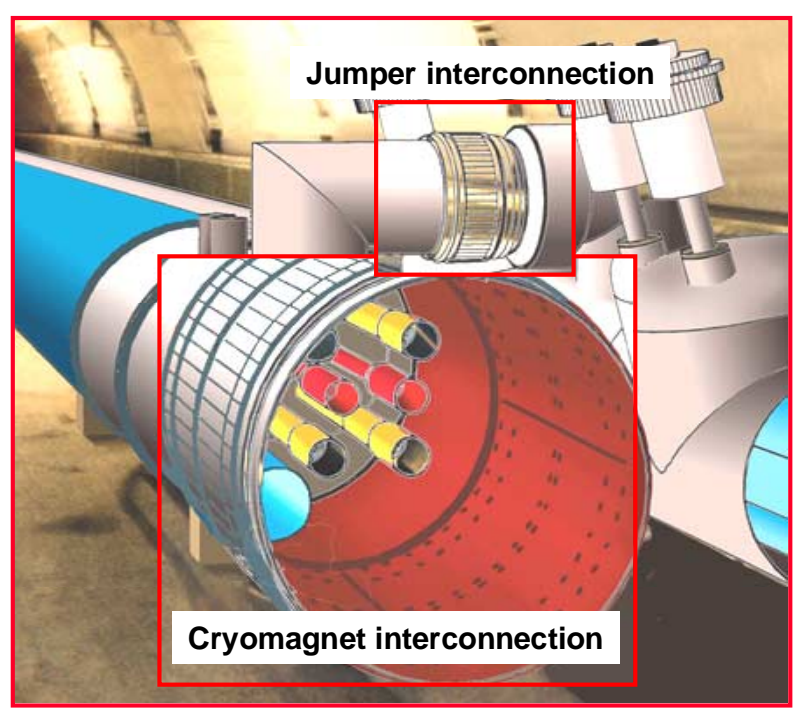

Figure 1: 3D view of the interconnections.

\section{JOINING TECHNOLOGIES}

In this chapter, the specific technologies used to assemble the LHC interconnection are described. They have to meet some general requirements:

- $\quad$ Limited space (longitudinally and radially).

- High reliability and quality.

- Economical and schedule constraints.

\subsection{Induction Soldering}

The main magnets are powered by Niobium/Titanium superconducting cables, carrying up to $13000 \mathrm{~A}$. Inside each magnet-to-magnet interconnection, six cables of this type must be joined. The main constraints and requirements applying to them are:

- $\quad$ High number of joints (about 10 000).

- High current intensity (up to $13000 \mathrm{~A}$ ).

- Low electrical resistance $(<0.6 \mathrm{n} \Omega$ to meet the requirements of the cryogenic budget).

Induction soldering was selected to perform a reliable junction of the main bus bars and to achieve a low electrical resistance at cold. To preserve the superconducting properties of the cables, the temperature and heating time have to be limited. Inductive heating permits a heating time of only about 90 seconds between $223{ }^{\circ} \mathrm{C}$ and $230{ }^{\circ} \mathrm{C}$. A non-corrosive flux was selected after intensive testing: mechanical, electrical, corrosion. To ensure a good quality of the soldered joint, the cable extremities must be in good condition (cleanliness, dimensional accuracy), and stabilized (pre-tinned) beforehand. This technology is giving very good results and has been used for STRING2 interconnections [1]. An attempt is presently made to render the process independent of the operator (closed loop control). 


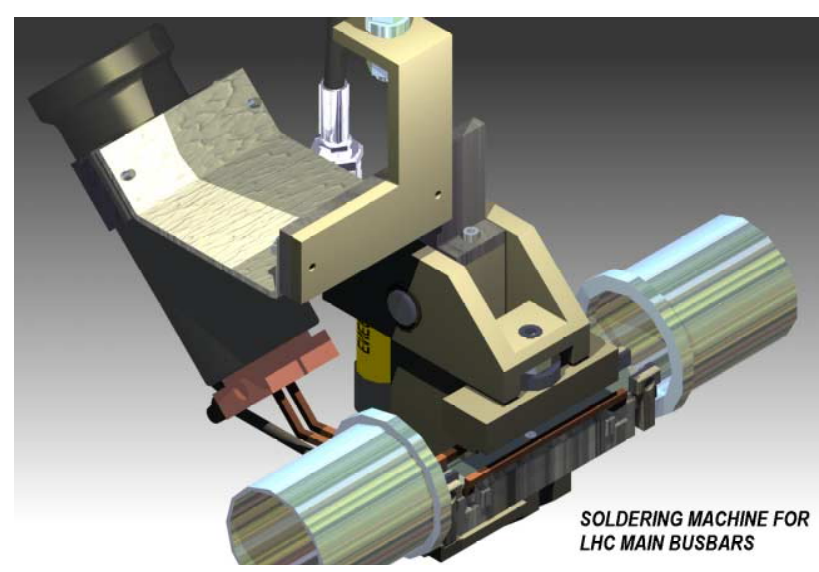

Figure 2: Induction soldering machine.

\subsection{Ultrasonic Welding}

This technology was developed in collaboration with LAPP (Laboratoire d'Annecy de Physique des Particules) to perform the junction of the auxiliary bus bars, [2]. The main constraints and requirements are:

- High number of joints (more than 50 000).

- Current intensity (up to $600 \mathrm{~A}$ ).

- Low electrical resistance $(<18 \mathrm{n} \Omega$ to meet the requirements of the cryogenic budget).

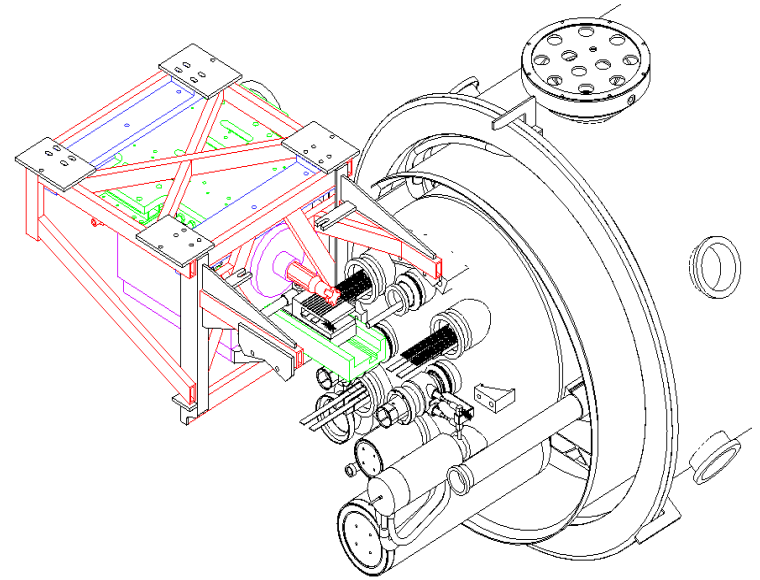

Figure 3: Ultrasonic welding machine.

The quality of the process is controlled by on-line recording of operating parameters such as power, driving in, time, dissipated energy. This technology was also applied for STRING2 and is giving very good results [1]. The average electrical resistance achieved is about $3 \mathrm{n} \Omega$.

\subsection{TIG Welding}

The very high number of welds (about 50000 ) to be performed in a very limited radial clearance of $45 \mathrm{~mm}$ requires a very reliable process.

The choice of TIG welding together with the automatic orbital machines associated with a specific geometry of the welds meets all these requirements. To ensure the reproducibility and quality, the on-line recording of the welding parameters (voltage, intensity, etc.) is foreseen.

\subsection{Orbital Cutting}

For testing and preparation activities, it is necessary to cut stainless steel pipes. Because of the very strict space constraints, it was necessary to accommodate the automatic orbital cutting machines with a $45 \mathrm{~mm}$ clearance. This technology could also be used for possible repair or upgrading activities.

\section{THE INTERCONNECTION ACTIVITIES}

\subsection{The Interconnection Assembly Sequences}

The sequence of operations necessary to carry out an interconnection has been defined following some general principles:

- From inside (beam lines) towards outside (vacuum vessel sleeve) for ease of work.

- Operations involving fragile components are carried out as late as possible.

- Whenever possible, operations requiring the same tooling are performed consecutively.

- The most delicate parts are protected (bellows, bus bars extremities, etc.).

- At some points, permission of those responsible for relevant systems is mandatory to go ahead.

\subsection{Interconnections between Cryomagnets}

After cold test and preparation for installation in the tunnel, the cryomagnets are transported in the tunnel and aligned. Then, the interconnection activities can start.

1. The RF modules are mounted. They provide smooth electrical continuity between adjacent beam screens [3]. They are TIG welded in-situ. (Fig. 4)

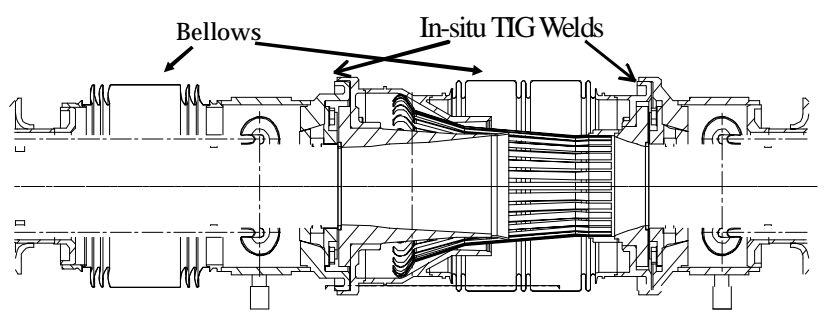

Figure 4: Beam lines interconnection.

2. The soldering of the six main bus bars is performed, applying the technology described in section 2.1. The electrical insulation is rebuilt. The 20 spool-piece bus bars are ultrasonically welded (see section 2.2).

3. An important electrical verification is performed. The main functions to check are: electrical continuity of circuits, electrical insulation (voltage withstand levels) and conformity to the electrical scheme.

4. The sleeves closing the cryogenic channels are slid in place and TIG welded.

5. The two heat exchanger lines are connected. The inner copper tube is soldered using a non-aggressive flux and the outer stainless steel sleeve is welded.

6. The support post cooling line is interconnected. This system is auto-stabilized thanks to compensation loops present at both sides.

7. The thermal shield is cooled by gaseous Helium [50 K to $65 \mathrm{~K}$ ]. The interconnection is realized by TIG welding of the expansion joint (Fig. 5). 


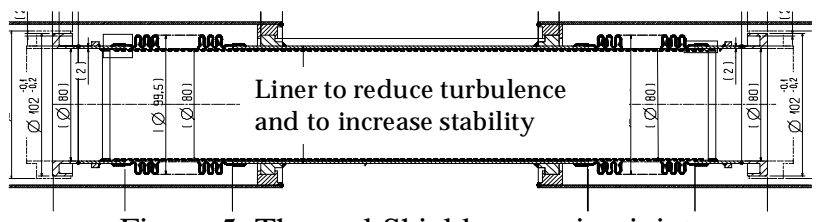

Figure 5: Thermal Shield expansion joint.

8. Metal hoses used to cool the beam screens [3] are then TIG welded.

9. Corrector magnets associated with main quadrupoles are powered via an auxiliary superconducting cable housed in an external line: the line $\mathrm{N}$. The superconducting cable is inserted in situ by portion of 54 meters. The superconducting bus bars are ultrasonically welded. The electrical connection scheme is varying with the interconnection location and its correctness is of vital importance because a mistake is very difficult to detect. The relevant connection boxes are closed.

10. After a careful cleaning of the interconnection zone, all the previously executed tasks are reviewed. This verification is based on checklists.

11. The radiative insulation and the thermal shields are mounted. They are composed of metal shells wrapped with MLI blankets.

12. The outside sleeve is closed using clamps and elastomer seals (Fig. 6).

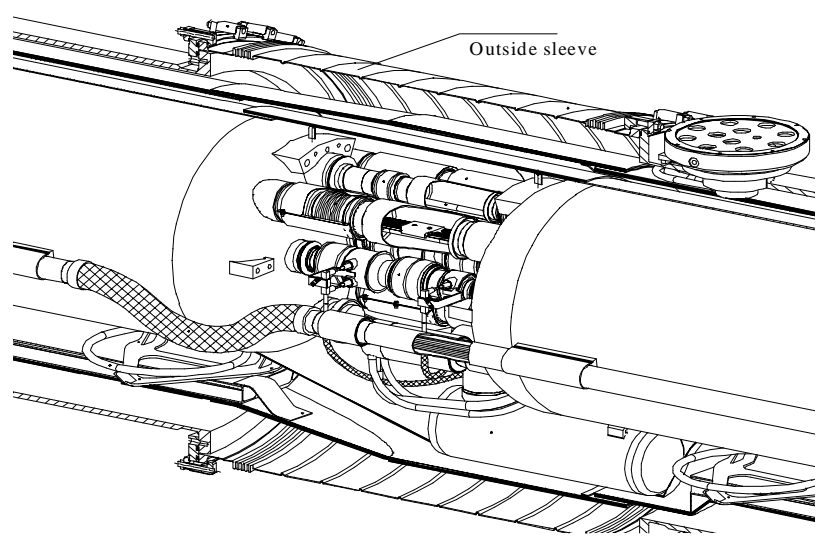

Figure 6: Interconnection between cryodipoles.

13. After completion of all activities, a formal review of all the aspects is organized.

\subsection{Jumper Interconnections}

About 250 junctions between cryomagnets and cryogenic distribution line have to be carried out (Fig. 7).

The technologies used are a subset of the ones previously described. After cutting and removing the end cap, the cryogenic channels are TIG welded. Then, similarly to the cryomagnets, the interconnection is cleaned and the radiative insulation and the thermal shields are mounted. Finally, the jumper outside sleeve is closed by two welds.

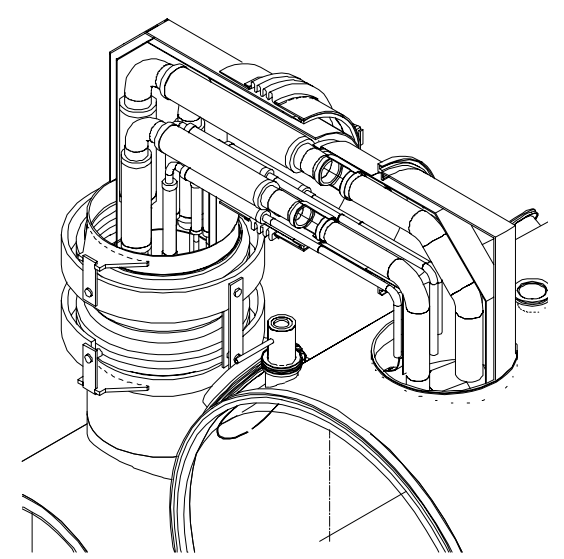

Figure 7: Jumper interconnection.

\section{QUALITY ASSURANCE ASPECTS}

A strict quality control will be implemented at different levels: component, process and procedure, sub-system and system. This can be summarized as follows:

- The components added during the interconnection are subjected to tests, both at manufacturer premises and at CERN in order to ensure the respect of the relevant technical specification.

- The critical processes are monitored on-line and the parameters must be in an acceptable range. In addition, to avoid long-term drift, samples are realized and extensively tested off-line. Detailed procedures are defined and their correct application is verified.

- At various steps, the persons responsible for subsystems are asked to make verifications to give their go-ahead for the continuation of the interconnection work. Check lists are used.

- For the interconnection, seen as a system, a formal review is organized upon completion of the activities. Its conclusion is to release the interconnection or to impose corrective actions.

\section{CONCLUSIONS}

The main technologies to be used for the LHC interconnections have been qualified during laboratory tests and were successfully applied for the STRING2 assembly. Work is now underway to industrialize the procedures and the tooling. The implementation of the quality control is in hand.

\section{ACKNOWLEDGMENTS}

The authors want to thank the LHC-CRI-I2 section and the LAPP for their support.

\section{REFERENCES}

[1] R. Saban et al., "The preparation of the cryomagnets and the assembly of the LHC Test String 2", proceed.

[2] J.C. Brunet et al., "An automatic ultrasonic welding process for interconnecting superconducting wires of the CERN Large Hadron Collider (LHC), ICEC 18, India 2000.

[3] R. Veness et al., "Beam vacuum interconnects for the LHC cold arcs", PAC 1999, New York City 